PROCEEDINGS OF THE

AMERICAN MATHEMATICAL SOCIETY

Volume 130, Number 7, Pages 2173-2177

S 0002-9939(02)06468-7

Article electronically published on February 4, 2002

\title{
LEFSCHETZ INDEX FOR ORIENTATION REVERSING PLANAR HOMEOMORPHISMS
}

\author{
MARC BONINO
}

(Communicated by Michael Handel)

\begin{abstract}
We prove that an isolated fixed point of an orientation reversing homeomorphism of the plane always has Lefschetz index 0 or \pm 1 .
\end{abstract}

\section{INTRODUCTION}

If $U$ is an open subset of $\mathbb{R}^{2}$ and $p \in U$ an isolated fixed point of a continuous map $h: U \rightarrow \mathbb{R}^{2}$, the Lefschetz index of $h$ at $p$, denoted by $\operatorname{Ind}(h, p)$, is the winding number of the vector field $h(z)-z$ on any simple closed curve surrounding $p$ and close enough to $p$. There is plentiful literature where this fixed-point index plays an important role; for example, to detect other fixed points, or to obtain local and/or global dynamical properties for surfaces homeomorphisms.

Throughout this paper, the map $h$ is a (local) homeomorphism and we will focus on the set of all the possible values for the Lefschetz index. It is well-known that, for every integer $n \in \mathbb{Z}$, there exists an orientation preserving planar homeomorphism $h_{n}$ which has the origin $o$ as an isolated fixed point and such that $\operatorname{Ind}\left(h_{n}, o\right)=n$ (see for example [1]). Surprisingly, M. Brown announced in the same paper that for an orientation reversing homeomorphism of the plane, the Lefschetz index is one of the three values $-1,0$ or +1 , but no proof of this result has been given until now. This motivated the writing of this paper, where we will show precisely the following:

Theorem. Let $V, W$ be two connected open subsets of $\mathbb{R}^{2}$ containing the origin $o$ and let $h: V \rightarrow W=h(V)$ be an orientation reversing homeomorphism which possesses $o$ as an isolated fixed point.

Then $\operatorname{Ind}(h, o) \in\{-1,0,+1\}$.

\section{INDEX ON A JORDAN CURVE}

For completeness, we recall briefly in this section some classical results and definitions.

If $C$ is a Jordan curve (i.e. a simple closed curve in $\mathbb{R}^{2}$, i.e. a subset of $\mathbb{R}^{2}$ homeomorphic to the unit circle $\mathbb{S}^{1}$ ), then $\mathbb{R}^{2} \backslash C$ has exactly two connected components and $C$ is their common frontier. The bounded one (resp. the unbounded one) is named the interior domain (resp. the exterior domain) of $C$ and is denoted by

Received by the editors February 2, 2001.

2000 Mathematics Subject Classification. Primary 55M20; Secondary 54H20.

(C)2002 American Mathematical Society 
$\operatorname{int}(C)$ (resp. by $\operatorname{ext}(C)$ ). A subset of $\mathbb{R}^{2}$ which is the interior domain of a Jordan curve is said to be a Jordan domain. In this paper, a Jordan curve $C$ is always counter-clockwise oriented. This yields an ordering relation (defined up to circular permutation) on $C$. If $x, y$ are two points on $C,[x, y]_{C}$ (resp. $\left.(x, y)_{C}\right)$ denotes the closed arc (resp. the open arc) on $C$ from $x$ to $y$ for this orientation of $C$.

Let $u: \mathbb{S}^{1} \rightarrow C$ be a homeomorphism which endows the Jordan curve $C=u\left(\mathbb{S}^{1}\right)$ with its counter-clockwise orientation. If $X$ is a subset of $\mathbb{R}^{2}$ containing $C$ and $f: X \rightarrow \mathbb{R}^{2}$ a continuous map without fixed point on $C$, the degree of the map $z \mapsto(f(u(z))-u(z)) /\|f(u(z))-u(z)\|\left(z \in \mathbb{S}^{1}\right)$ does not depend on the choice of $u$. It is named the index of $f$ on $C$ and is denoted by $\operatorname{Ind}(f, C)$. Now, if $X$ is open and $p \in X$ is an isolated fixed point of $f$, choose a disk neighborhood $D$ of $p$, so small that $D \subset X$ and $f$ has no other fixed point in $D$. Then the index of $f$ is the same on any Jordan curve $C$ lying in $D$ and such that $p \in \operatorname{int}(C)$, and this common value defines the Lefschetz index $\operatorname{Ind}(f, p)$ of $f$ at the point $p$.

\section{Proof of the Theorem}

In the following, we will write respectively $\bar{X}$ and $\partial X$ for the closure and the frontier of a set $X \subset \mathbb{R}^{2}$.

Choose once and for all a circle $C$ around $o$, so that the closed disk $D=\overline{\operatorname{int}(C)}$ is contained in $V \cap W$ and $o$ is the only fixed point of $h$ in $D$. First, observe there are two cases where the index can be easily computed:

- if $h(D) \subset D$, then $\operatorname{Ind}(h, o)=1$,

- if $D \subset h(D)$, then, since $h$ reverses the orientation, $\operatorname{Ind}(h, o)=-\operatorname{Ind}\left(h^{-1}, o\right)$ $=-1$.

From now on, we exclude these two simple situations, that is we suppose $h(\operatorname{int}(C))$ $\not \subset \operatorname{int}(C)$ and $\operatorname{int}(C) \not \subset h(\operatorname{int}(C))$.

It is well known since Kerékjártó that every connected component of the intersection of two Jordan domains is again a Jordan domain. We need a more precise description, which can be stated as follows. All the assertions in Proposition 3.1 and their proofs are contained in the first section of [2].

Proposition 3.1. Let $U, U^{\prime}$ be two Jordan domains containing the origin o, such that $U \not \subset U^{\prime}$ and $U^{\prime} \not \subset U$. Denote by $U \wedge U^{\prime}$ the connected component of $U \cap U^{\prime}$ which contains $o$ and by $\partial U \wedge \partial U^{\prime}$ the frontier of $U \wedge U^{\prime}$.

(1) We have a partition

$$
\partial U \wedge \partial U^{\prime}=\left(\left(\partial U \wedge \partial U^{\prime}\right) \cap \partial U \cap \partial U^{\prime}\right) \bigcup_{i \in I} \alpha_{i} \bigcup_{j \in J} \beta_{j}
$$

where

- $I, J$ are nonempty and at most countable sets,

- for every $i \in I, \alpha_{i}=\left(a_{i}, b_{i}\right)_{\partial U}$ is a connected component of $\partial U \cap U^{\prime}$,

- for every $j \in J, \beta_{j}=\left(c_{j}, d_{j}\right)_{\partial U^{\prime}}$ is a connected component of $\partial U^{\prime} \cap U$.

(2) For every $j \in J, \quad U \wedge U^{\prime}$ is contained in the Jordan domain bounded by $\beta_{j} \cup\left[d_{j}, c_{j}\right]_{\partial U}$.

(3) $\partial U \wedge \partial U^{\prime}$ is homeomorphic to $\partial U$. In particular, it is a Jordan curve.

(4) Three points a, b, c of $\left(\partial U \wedge \partial U^{\prime}\right) \cap \partial U$ (resp. of $\left.\left(\partial U \wedge \partial U^{\prime}\right) \cap \partial U^{\prime}\right)$ are met in this order on $\partial U$ (resp on $\left.\partial U^{\prime}\right)$ if and only if they are met in the same order on $\partial U \wedge \partial U^{\prime}$. 


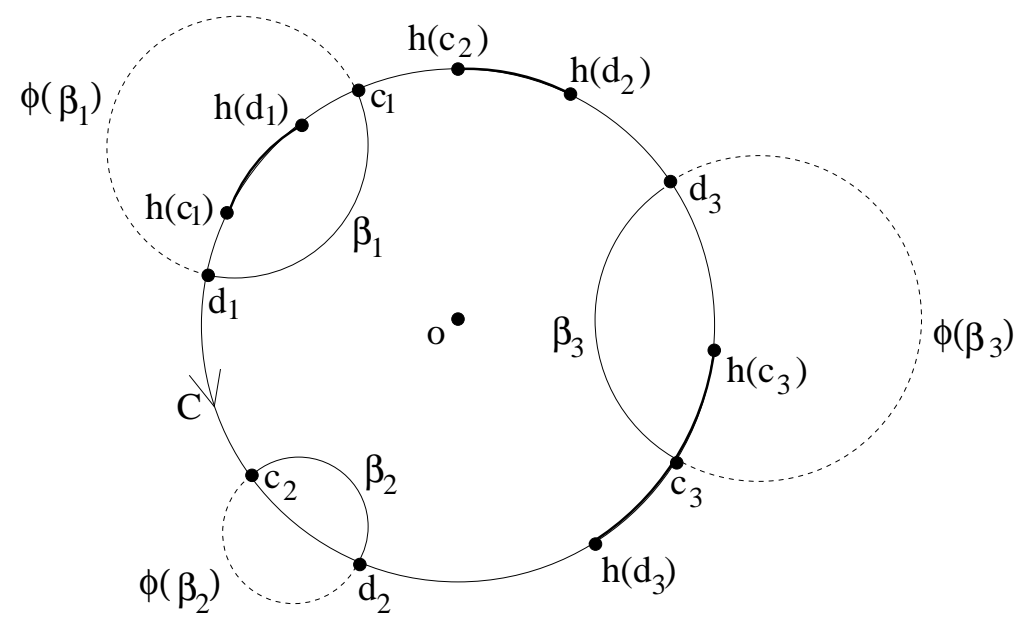

Figure 1. The curves $\Gamma$ and $\phi(\Gamma)$

Let us consider the partition $\left(P_{h}\right)$ and the $\operatorname{arcs} \alpha_{i}$ and $\beta_{j}$ that we obtain when we apply Proposition 3.1 with $U=\operatorname{int}(C)$ and $U^{\prime}=\operatorname{int}\left(h^{-1}(C)\right)=h^{-1}(U)$.

Let $\phi$ be the inversion in the circle $C$ and $\Gamma=C \wedge h^{-1}(C)$ the Jordan curve given by Proposition 3.1 Since $\phi$ is a homeomorphism of $\mathbb{R}^{2} \backslash\{o\}, \phi(\Gamma)$ is also a Jordan curve.

Now, define a map $H$ from $\Gamma \cup \phi(\Gamma)$ to $\mathbb{R}^{2}$ by setting

$$
H(z)= \begin{cases}h(z) & \text { if } z \in \Gamma \\ h(\phi(z)) & \text { if } z \in \phi(\Gamma) .\end{cases}
$$

Clearly, $H$ is well-defined, continuous and fixed-point free. Since $H(\phi(\Gamma))=h(\Gamma) \subset$ $D \subset \overline{\operatorname{int}(\phi(\Gamma))}$, we have $\operatorname{Ind}(H, \phi(\Gamma))=1$. We have also $H=h$ on $\Gamma$ and consequently $\operatorname{Ind}(H, \Gamma)=\operatorname{Ind}(h, \Gamma)=\operatorname{Ind}(h, o)$.

Observe that, for every $j \in J$, the set $C_{j}=\overline{\beta_{j}} \cup \phi\left(\overline{\beta_{j}}\right)$ is a Jordan curve such that $\left(c_{j}, d_{j}\right)_{C} \subset \operatorname{int}\left(C_{j}\right)$ and $\left(d_{j}, c_{j}\right)_{C} \subset \operatorname{ext}\left(C_{j}\right)$. The orientations induced on $\beta_{j}$ by $C_{j}$ and $\Gamma$ are opposite (see Figure 1 ). Furthermore, the $\beta_{j}$ are pairwise disjoint open $\operatorname{arcs}$ in $h^{-1}(C)$ and then, for a given $\epsilon>0$, there is only a finite number of indexes $j \in J$ such that the diameter of $\beta_{j}$ is superior to $\epsilon$. It follows that there are only finitely many indexes $j \in J$ such that $\operatorname{Ind}\left(H, C_{j}\right) \neq 0$.

Bringing together the above remarks, we obtain the formula

$$
1=\operatorname{Ind}(H, \phi(\Gamma))=\operatorname{Ind}(h, 0)+\sum_{j \in J} \operatorname{Ind}\left(H, C_{j}\right) .
$$

Keeping in mind that, for every $j \in J$, we have $H\left(C_{j}\right)=h\left(\overline{\beta_{j}}\right)=\left[h\left(d_{j}\right), h\left(c_{j}\right)\right]_{C}$, we now state three basic lemmas:

Lemma 3.2. If $\left[h\left(d_{j}\right), h\left(c_{j}\right)\right]_{C} \cap\left[c_{j}, d_{j}\right]_{C}=\emptyset$, then $\operatorname{Ind}\left(H, C_{j}\right)=0$.

Proof. It is enough to remark that $H\left(C_{j}\right)=\left[h\left(d_{j}\right), h\left(c_{j}\right)\right]_{C} \subset\left(d_{j}, c_{j}\right)_{C} \subset \operatorname{ext}\left(C_{j}\right)$.

Lemma 3.3. If the set $\left[h\left(d_{j}\right), h\left(c_{j}\right)\right]_{C} \cap\left[c_{j}, d_{j}\right]_{C}$ is nonempty and connected, then $\operatorname{Ind}\left(H, C_{j}\right)=1$. 
Proof. This is clear if $\left[h\left(d_{j}\right), h\left(c_{j}\right)\right]_{C} \subset\left[c_{j}, d_{j}\right]_{C}$ because we have then $H\left(C_{j}\right) \subset$ $\overline{\operatorname{int}\left(C_{j}\right)}$. The following argument allows us to be reduced to this case: Denote $\left[z_{1}, z_{2}\right]_{C}=\left[h\left(d_{j}\right), h\left(c_{j}\right)\right]_{C} \cap\left[c_{j}, d_{j}\right]_{C}$. Let $\left(r_{t}\right)_{0 \leq t \leq 1}$ be a strong retracting deformation of the arc $\left[h\left(d_{j}\right), h\left(c_{j}\right)\right]_{C}$ onto its subarc $\left[z_{1}, z_{2}\right]_{C}$. The maps $\left.r_{t} \circ H\right|_{C_{j}}$ : $C_{j} \rightarrow \mathbb{R}^{2}(0 \leq t \leq 1)$ are fixed-point free because $C_{j} \cap r_{t}\left(H\left(C_{j}\right)\right) \subset\left\{c_{j}, d_{j}\right\}$ and, if $H\left(c_{j}\right)=h\left(c_{j}\right) \notin\left[z_{1}, z_{2}\right]_{C}$ (resp. if $\left.H\left(d_{j}\right)=h\left(d_{j}\right) \notin\left[z_{1}, z_{2}\right]_{C}\right)$, then $\left[z_{2}, h\left(c_{j}\right)\right]_{C}=$ $\left[d_{j}, h\left(c_{j}\right)\right]_{C}$ does not contain the point $c_{j}$ (resp. $\left[h\left(d_{j}\right), z_{1}\right]_{C}=\left[h\left(d_{j}\right), c_{j}\right]_{C}$ does not contain the point $\left.d_{j}\right)$. Since the map $t \mapsto \operatorname{Ind}\left(\left.r_{t} \circ H\right|_{C_{j}}, C_{j}\right)$ is continuous and $r_{1}\left(H\left(C_{j}\right)\right)=\left[z_{1}, z_{2}\right]_{C} \subset\left[c_{j}, d_{j}\right]_{C} \subset \overline{\operatorname{int}\left(C_{j}\right)}$, we obtain $\operatorname{Ind}\left(H, C_{j}\right)=$ $\operatorname{Ind}\left(\left.r_{1} \circ H\right|_{C_{j}}, C_{j}\right)=1$.

Lemma 3.4. There are at most two indexes $j \in J$ such that $\left[h\left(d_{j}\right), h\left(c_{j}\right)\right]_{C} \cap$ $\left[c_{j}, d_{j}\right]_{C}$ is nonempty.

Proof. Suppose that we can find three distinct arcs $\beta_{j_{k}}(k \in\{1,2,3\})$ among the $\beta_{j}$ such that $\left[h\left(d_{j_{k}}\right), h\left(c_{j_{k}}\right)\right]_{C} \cap\left[c_{j_{k}}, d_{j_{k}}\right]_{C} \neq \emptyset$. Since $h$ has no fixed point on $C$, it is the same to write $\left(h\left(d_{j_{k}}\right), h\left(c_{j_{k}}\right)\right)_{C} \cap\left(c_{j_{k}}, d_{j_{k}}\right)_{C} \neq \emptyset$ and then one can choose a point $x_{k} \in \beta j_{k}$ such that $h\left(x_{k}\right) \in\left(c_{j_{k}}, d_{j_{k}}\right)_{C}$. Renaming the indexes $j_{k}$ if necessary, one can suppose that $\beta_{j_{1}}, \beta_{j_{2}}, \beta_{j_{3}}$ are met in this order on $h^{-1}(C)$.

On one hand, since $x_{k} \in \beta j_{k}$ and $h$ reverses the orientation, we must have $h\left(x_{3}\right), h\left(x_{2}\right), h\left(x_{1}\right)$ in this order on $C$. On the other hand, we know from Proposition 3.1 (4) that the points $c_{j_{1}}, d_{j_{1}}, c_{j_{2}}, d_{j_{2}}, c_{j_{3}}, d_{j_{3}}$ are met in this order on $h^{-1}(C)$, on $\Gamma$ and on $C$. With $h\left(x_{k}\right) \in\left(c_{j_{k}}, d_{j_{k}}\right)_{C}$, we obtain now that $h\left(x_{1}\right), h\left(x_{2}\right), h\left(x_{3}\right)$ are in this order on $C$. This is not possible because the points $h\left(x_{k}\right)(k \in\{1,2,3\})$ are pairwise distinct.

We can now complete the proof of the Theorem: If, for every $j \in J$, the set $\left[h\left(d_{j}\right), h\left(c_{j}\right)\right]_{C} \cap\left[c_{j}, d_{j}\right]_{C}$ is either empty or connected, the result is an obvious consequence of formula $(*)$ and of Lemmas (3.2)-3.4).

Otherwise, there exists an arc $\beta=(c, d)_{h^{-1}(C)}$ among the $\beta_{j}$ such that $c, h(c)$, $h(d), d$ are met in this order on $C$. According to Proposition 3.1 (2) and (4), the points $d, a_{i}, b_{i}, c$ are met in this order on $C$ and on $h^{-1}(C)$ for every $i \in I$. It follows that $c, h(c), h\left(b_{i}\right), h\left(a_{i}\right), h(d), d$ are in this order on $C$. Thus, we obtain $\overline{\alpha_{i}} \cap\left[h\left(b_{i}\right), h\left(a_{i}\right)\right]_{C}=\emptyset$. For convenience, let us define now $g=h^{-1}$. Of course, one can use Proposition 3.1 with $U=\operatorname{int}(C)$ and $U^{\prime}=\operatorname{int}\left(g^{-1}(C)\right)$ and then obtain a corresponding partition $\left(P_{g}\right)$ for $C \wedge g^{-1}(C)$. But $C \wedge g^{-1}(C)$ is nothing but $h\left(C \wedge h^{-1}(C)\right)$ and therefore the partition $\left(P_{g}\right)$ can be written using the $\operatorname{arcs} \alpha_{i}$ and $\beta_{j}$ of $\left(P_{h}\right)$. Precisely:

$$
C \wedge g^{-1}(C)=\left(\left(C \wedge g^{-1}(C)\right) \cap C \cap g^{-1}(C)\right) \bigcup_{j \in J} h\left(\beta_{j}\right) \bigcup_{i \in I} h\left(\alpha_{i}\right) .
$$

Observe that the $\operatorname{arcs} h\left(\alpha_{i}\right)=\left(h\left(b_{i}\right), h\left(a_{i}\right)\right)_{g^{-1}(C)}$ play the same role in $\left(P_{g}\right)$ as the $\operatorname{arcs} \beta_{j}$ in $\left(P_{h}\right)$. Since $\overline{\alpha_{i}}=g\left(h\left(\overline{\alpha_{i}}\right)\right)$ is disjoint from $\left[h\left(b_{i}\right), h\left(a_{i}\right)\right]_{C}$ for every $i \in I$, we see that we are reduced to the previous situation if we replace $h$ by $g$. Then we obtain $\operatorname{Ind}(h, o)=-\operatorname{Ind}(g, o)=-1$. The Theorem is proved.

\section{ACKNOWLEDGEMENTS}

I would like to thank P. Le Calvez, who improved a preliminary version of this paper. 


\section{REFERENCES}

[1] M. Brown, On the fixed points index of iterates of planar homeomorphisms, Proc. Amer. Math. Soc. 108 (1990), 1109-1114. MR 90g:54036

[2] P. Le Calvez and J.C. Yoccoz, Un théorème d'indice pour les homéomorphismes du plan au voisinage d'un point fixe, Annals of Mathematics 146 (1997), 241-293. MR 99a:58129

Université Paris 13, Institut Galilée, Département de Mathématiques, Avenue J.B. Clément, 93430 Villetaneuse, France

E-mail address: bonino@math.univ-paris13.fr 